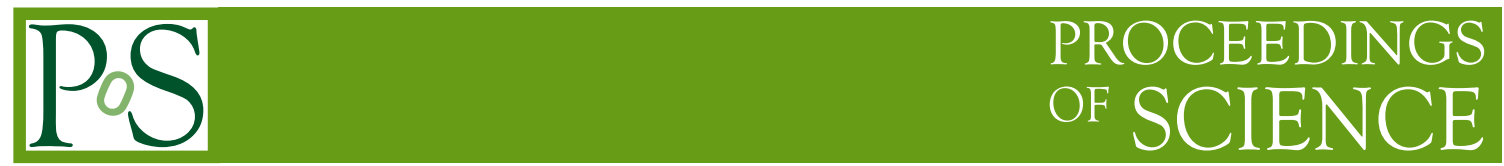

\title{
Probing dark matter with ILC
}

\section{Wojciech Kotlarski ${ }^{a, *}$ and Aleksander Filip Żarnecki ${ }^{b}$ on behalf of the International Linear Collider International Development Team Physics and Detector Working Group}

${ }^{a}$ Institut für Kern- und Teilchenphysik,

Zellescher Weg 19, 01069 Dresden, Germany

${ }^{b}$ Faculty of Physics, University of Warsaw,

Pasteura 5, 02-093 Warsaw, Poland

E-mail: wojciech.kotlarski@tu-dresden.de, filip.zarnecki@fuw.edu.pl

The International Linear Collider (ILC) offers a number of unique opportunities for searches for dark matter and dark sector particles. The collider program itself will offer important capabilities, including precision measurements in the scalar sector and direct searches based on the monophoton signature. With extreme intensities of the high-energy electron and positron beams, the ILC will also enable new fixed-target measurements, both beam dump experiments and dedicated experiments using extracted beams. Described in this contribution are the expectations for these programs, which address all of the possible dark sector portals.

*** The European Physical Society Conference on High Energy Physics (EPS-HEP2021), ***

*** 26-30 July $2021 * * *$

*** Online conference, jointly organized by Universität Hamburg and the research center DESY ***

${ }^{*}$ Speaker 


\section{Introduction}

While there are many hints for existence of Dark Matter (DM), its nature is basically not known. Many possible scenarios need to be considered with different possible connections between Standard Model (SM) and DM sectors, a wide range of DM particle masses, coupling values and other model parameters. The ILC, with its high energy reach, clean environment and kinematic constraints of $\mathrm{e}^{+} \mathrm{e}^{-}$collisions, is a unique machine offering many options for DM searches. Additional capabilities open with the use of single high-intensity electron or positron beams in a fixed target mode.

The ILC will make use of the superconducting accelerating cavities. In the assumed staged construction scheme, it will start as a $250 \mathrm{GeV}$ Higgs factory, followed by the $500 \mathrm{GeV}$ machine, with a possible upgrade even to $1 \mathrm{TeV}$. The unique feature of the ILC is the possibility of having both electron and positron beams polarised. This is crucial for many precision measurements as well as for beyond SM searches. By combining data collected with four different polarisation combinations accuracy of precision measurements is significantly increased, more constraints can be imposed in global fits and analyses, ambiguity between different scenarios can be removed in many BSM studies and the sensitivity to systematic effects can be significantly reduced.

\section{Higgs measurements}

The first ILC running stage will clearly focus on the Higgs boson measurements. At $250 \mathrm{GeV}$ its production is dominated by the Higgs-strahlung process, $\mathrm{e}^{+} \mathrm{e}^{-} \rightarrow Z H$. In this channel the so called "Z-tagging" method can be used for unbiased selection of Higgs production events by reconstructing the recoil mass. This allows for fully model independent analysis avoiding any dependence on the Higgs decay channel. The recoil mass technique results also in high sensitivity to invisible Higgs boson decays $[1,2]$. For $2 \mathrm{ab}^{-1}$ collected at $250 \mathrm{GeV}$ ILC the expected $95 \%$ C.L. limit on the invisible Higgs boson decays is $0.23 \%$ [1], an order of magnitude better than the estimated HL-LHC sensitivity. The expected recoil mass distribution for the ILC running at $250 \mathrm{GeV}$, assuming an invisible Higgs boson branching ratio of 10\%, is shown in Fig. 1 (left).

In Higgs-portal models, new scalar fields coupled to DM particles can mix with the SM Higgs field. If the mixing angle $\theta$ is small, one of the mass eigenstates is SM-like (the observed $125 \mathrm{GeV}$
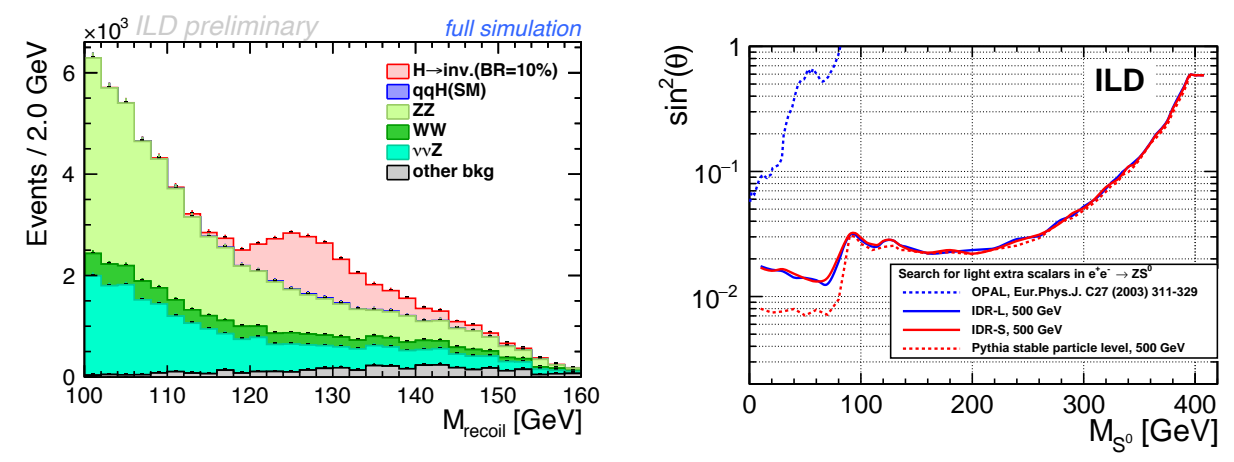

Figure 1: Left: recoil mass distribution after event selection for ILC running at $250 \mathrm{GeV}$ and invisible Higgs branching ratio of $10 \%$ [1]. Right: expected sensitivity of ILC running at $500 \mathrm{GeV}$ to the scalar sector mixing parameter $\sin ^{2} \theta[3]$. 
scalar). If the second mass eigenstate is also light, it can be produced in $\mathrm{e}^{+} \mathrm{e}^{-}$collisions in the same way as the SM-like Higgs boson and should be visible as an additional resonance in the recoil mass distribution. Such scenarios can be probed at the ILC down to $\sin ^{2} \theta \sim 10^{-2}$, see Fig. 1 (right) [3].

\section{Mono-photon events}

DM particles can be pair produced in the $\mathrm{e}^{+} \mathrm{e}^{-}$collisions via exchange of a new mediator particle, which couples to both electrons and DM particles, $\chi$. This process can be detected, if additional hard photon radiated from the initial state, $\mathrm{e}^{+} \mathrm{e}^{-} \rightarrow \chi \chi+\gamma$, is observed in the detector. The mono-photon signature is considered to be the most general approach to search for DM particle production at $\mathrm{e}^{+} \mathrm{e}^{-}$colliders, as it is largely independent of the BSM scenario. Signal events have a striking signature: single high-energy photon and no other activity in the detector. "Irreducible" background in this channel comes from the radiative neutrino pair-production, $\mathrm{e}^{+} \mathrm{e}^{-} \rightarrow v \bar{v}+\gamma$, while huge background from the radiative Bhabha scattering, $\mathrm{e}^{+} \mathrm{e}^{-} \rightarrow \mathrm{e}^{+} \mathrm{e}^{-}+\gamma$, can be efficiently suppressed by the selection cuts.

Scenarios with DM pair-production via heavy mediator exchange were considered in a full simulation study [4], using an EFT approach and assuming coupling values $O(1)$. For light DM particles mediator masses up to about $3 \mathrm{TeV}$ can be excluded at the ILC, as shown in Fig. 2 (left). DM production via light mediator exchange is still not excluded if very small mediator couplings to SM particles (electrons) are considered. Most general, "experimental-like" approach to such scenarios was presented in [5], where limits on the DM pair-production were studied as a function of mediator mass and width. Dedicated simulation procedure was used [6], with all "detectable" photons generated on a Matrix Element level, matched with soft ISR. With 2-dim distribution of $\left(p_{T}^{\gamma}, \eta^{\gamma}\right)$ used to constrain DM production, ILC running at $500 \mathrm{GeV}$ is sensitive to mediator couplings down to $O\left(10^{-3}\right)$, as shown in Fig. 2 (right).

\section{Non-collider experiments}

Extreme intensities of electron and positron beams at the ILC, $O\left(10^{22}\right)$ particles per year, open unique options for fixed-target experiments focused on rare processes. General concept of main
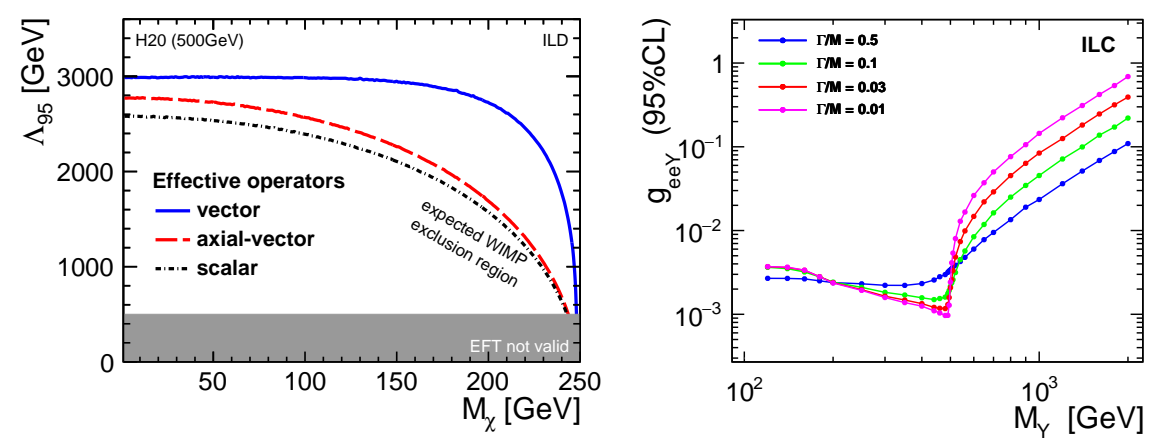

Figure 2: The expected sensitivity of ILC running at $500 \mathrm{GeV}$ to DM pair production. Left: EFT limits on mediator mass, as a function of the DM particle mass, for different mediator types [4]. Right: mediator coupling limits as a function of the vector mediator mass and DM particle mass of $50 \mathrm{GeV}$ [5]. 
beam dump experiments searching for axion-like particles or new scalars is presented in Fig. 3 [7]. Light axion-like particles ( $a$ ) could be copiously produced in the beam dump and decay only after passing the massive muon shield. Expected sensitivity of ILC running at $250 \mathrm{GeV}$ to $a \gamma \gamma$ coupling as a function of its mass, resulting from the search for two-photon decay, $a \rightarrow \gamma \gamma$, is presented in Fig. 4 (left). Thanks to the extreme beam intensities the ILC sensitivity is an order of magnitude better than those expected at other experiments. Similar increase in sensitivity is expected for models with new light scalars coupling to charged leptons. Expected sensitivity of the ILC to the scenario with scalar coupling proportional to the lepton mass is shown in Fig. 4 (right).

Other options for light DM particle searches in fixed-target experiments at the ILC were considered in [8]. If DM particles are light, dark photon $\mathrm{A}^{\prime}$ production in the beam dump could result in a collimated stream of $\mathrm{DM} \chi$ particles from $\mathrm{A}^{\prime}$ decay, $\mathrm{A}^{\prime} \rightarrow \chi \chi$, which can be probed by looking for elastic DM interactions in the dedicated detector. This approach, previously used in SLAC Beam Dump Experiment E137 [9], will offer huge improvement in sensitivity for $m_{A^{\prime}} \lesssim 1 \mathrm{GeV}$ thanks to much higher statistics at the ILC. Experiments with extracted positron beams can search for dark photon production independent on its decays. Associated production, $\mathrm{e}^{+} \mathrm{e}^{-} \rightarrow \mathrm{A}^{\prime} \gamma$ can be searched for with thick active target reconstructing the missing energy or in thin target detectors reconstructing the missing mass. In both approaches, sensitivity of the fixed-target experiments at the ILC can probe the dark photon couplings down to the minimum couplings allowed by relic

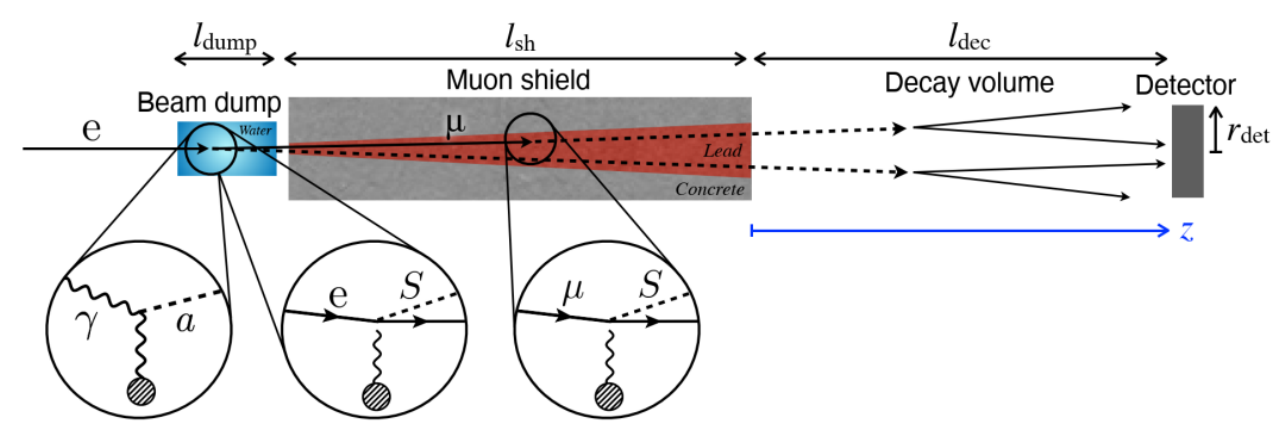

Figure 3: An experimental setup proposed for new light particle searches at the ILC [7].
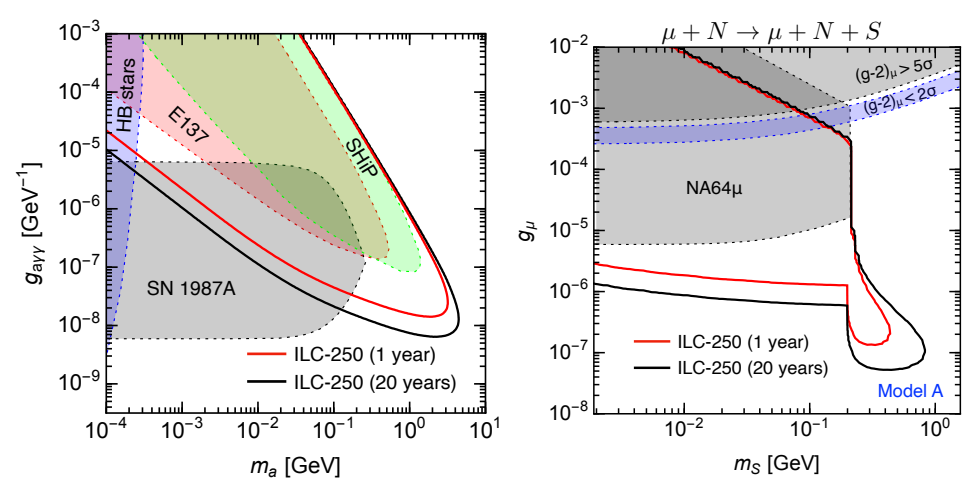

Figure 4: Expected sensitivity of main beam dump experiments at the $250 \mathrm{GeV}$ ILC after one year (red lines) and 20 years (black lines) of running to processes with light axion-like particle production (left) and light scalar production (right). Results taken from [7]. 
density bounds.

\section{Conclusions}

ILC will offer many complementary options for DM searches. Different scenarios can be constrained via precision Higgs studies. Direct searches for DM pair-production are possible in the mono-photon channel with sensitivity to heavy mediator exchange reaching $\mathrm{TeV}$ mass scales, order of magnitude higher than the collision energy, and sensitivity to couplings of $O\left(10^{-3}\right)$ in the case of light mediator exchange. The ILC will also offer highest energy electron and positron beams, with unprecedented intensities, for beam dump and extracted beam experiments. Fixed-target experiments offer many interesting opportunities for dark sector searches in the low mass domain and other science goals.

Acknowledgments: Work supported by the National Science Centre, Poland, the OPUS project UMO-2017/25/B/ST2/00496 (2018-2021) and the HARMONIA project UMO-2015/18/M/ ST2/00518 (2016-2021).

\section{References}

[1] Y. Kato, Probing the dark sector via searches for invisible decays of the Higgs boson at the ILC, PoS EPS-HEP2019 (2020) 358 [2002 . 12048].

[2] A. Steinhebel, J. Brau and C. Potter, $H \rightarrow$ invisible at the ILC with SiD, in International Workshop on Future Linear Colliders, 4, 2021 [2105. 00128].

[3] Y. Wang, M. Berggren and J. List, ILD Benchmark: Search for Extra Scalars Produced in Association with a $Z$ boson at $\sqrt{s}=500 \mathrm{GeV}, 2005.06265$.

[4] M. Habermehl, M. Berggren and J. List, WIMP Dark Matter at the International Linear Collider, Phys. Rev. D 101 (2020) 075053 [2001.03011].

[5] J. Kalinowski, W. Kotlarski, K. Mekala, P. Sopicki and A.F. Zarnecki, Sensitivity of future $e^{+} e^{-}$colliders to processes of dark matter production with light mediator exchange, 2107.11194.

[6] J. Kalinowski, W. Kotlarski, P. Sopicki and A.F. Zarnecki, Simulating hard photon production with WHIZARD, Eur. Phys. J. C 80 (2020) 634 [2004 . 14486].

[7] Y. Sakaki and D. Ueda, Searching for new light particles at the international linear collider main beam dump, Phys. Rev. D 103 (2021) 035024 [2009. 13790].

[8] M. Perelstein, First estimates of the dark sector capabilities at ILC, presented at the 2021 International Workshop on Future Linear Colliders (LCWS2021), link to contribution.

[9] B. Batell, R. Essig and Z. Surujon, Strong Constraints on Sub-GeV Dark Sectors from SLAC Beam Dump E137, Phys. Rev. Lett. 113 (2014) 171802 [1406. 2698]. 